\title{
Thermal noise from icy mirrors in gravitational wave detectors
}

\author{
Jessica Steinlechner $\circledast^{1,2}$ and Iain W. Martin $\oplus^{2, *}$ \\ ${ }^{1}$ Institut für Laserphysik und Zentrum für Optische Quantentechnologien, Universität Hamburg, \\ Luruper Chaussee 149, 22761 Hamburg, Germany \\ ${ }^{2}$ SUPA, School of Physics and Astronomy, University of Glasgow, Glasgow G12 8QQ, Scotland
}

(Received 24 June 2019; published 12 August 2019)

\begin{abstract}
The detection of gravitational waves has established a new and very exciting field of astronomy in the past few years. To increase the number of detections and allow observation of a wider range of sources, several future gravitational wave detectors will operate at cryogenic temperatures. Recent investigations of a mirror in one of the cryostats of the Japanese KAGRA detector showed a decrease in reflectivity due to ice growth, induced by residual water molecules moving from the warm to the cold sections of the detector's vacuum system. Based on the optical measurements made in KAGRA, in this paper we calculate the implications of an ice layer on coating thermal noise for the planned European Einstein Telescope. We find coating thermal noise to oscillate, due to periodic reflectivity changes as the ice layer grows. The average coating thermal noise increases significantly over a time of one year with a larger increase at higher temperatures.
\end{abstract}

DOI: 10.1103/PhysRevResearch.1.013008

\section{INTRODUCTION}

The 14th of September 2015 marks the ground-breaking event of the first detection of gravitational waves [1], almost exactly 100 years after Einstein predicted their existence [2,3]. Since then, nine more gravitational wave signals from binary black-hole mergers and one from a binary neutron-star merger have been detected [4-8] by the Advanced LIGO [9] gravitational wave detectors during their first two observing runs. The Advanced Virgo detector [10] started observing during the second run and contributed to 5 of the 11 detections [8].

Gravitational wave observatories are interferometers with perpendicular arms several kilometers in length. Their hearts are formed by large-scale, highly reflective (HR) coated mirrors. An HR coating is made of a stack of layers of two (or more) materials with alternating high and low refractive index $n$. The reflectivity increases with the refractive-index difference of the materials and with the number of layers. For a given number of layers, the reflectivity maximizes for an optical thickness of $n \times d_{j}=\lambda / 4$ for each layer, in which $d_{j}$ is the geometric thickness of the $j$ th layer and $\lambda$ the wavelength at which the coating is designed to be reflective. The HR coated mirrors at the ends of the gravitational wave detector arms (end test masses, ETMs) have reflectivities of about $99.9995 \%$.

Once the advanced observatories reach their design sensitivity, coating thermal noise (CTN) from these mirrors will limit our ability to detect more gravitational waves from

\footnotetext{
*iain.martin@glasgow.ac.uk

Published by the American Physical Society under the terms of the Creative Commons Attribution 4.0 International license. Further distribution of this work must maintain attribution to the author(s) and the published article's title, journal citation, and DOI.
}

more distant or weaker sources. The magnitude of the CTN amplitude spectral density, to which a gravitational wave detector is sensitive, can be approximated by

$$
x(f)=\sqrt{\frac{2 k_{B} T}{\pi^{2} f} \frac{d}{w^{2}} \phi\left(\frac{Y_{\text {coat }}}{Y_{\text {sub }}^{2}}+\frac{1}{Y_{\text {coat }}}\right)} .
$$

Here, $k_{B}$ is the Boltzmann constant, $T$ the mirror temperature, $f$ the frequency, $d=\sum_{j} d_{j}$ the coating thickness, $w$ the radius of the laser beam on the coating, and $\phi$ the mechanical loss of the coating. $Y_{\text {sub }}$ and $Y_{\text {coat }}$ are the Young's moduli of the substrate and coating materials. Following Yam et al. [11] we assume, in this formula, that the mechanical losses associated with bulk motion and shear motion [12] are approximately equal ( $\left.\phi_{\text {bulk }} \approx \phi_{\text {shear }} \approx \phi\right)$. We follow the common convention in thermal noise modeling and assume that the mechanical loss is independent of frequency (i.e., structural damping). While the loss of amorphous coatings is not always frequency independent, the magnitude of any frequency dependence is usually small [13], and this is a good approximation for many materials [14].

Further, for simplicity, Eq. (1) neglects the effect of field penetration into the coating stack, and assumes that the Poisson's ratios $\sigma$ of the coating and the substrate are $0 .{ }^{1}$ The resulting formula [Eq. (1)] is identical to the previous description by Harry et al. [15] under similar approximations, and is often used for estimating CTN. However, for the CTN discussions made later in this paper, we used the more complete model by Yam et al. [11], which considers the different contribution of layers at different depths in the coating stack and does not neglect the Poisson ratios.

From Eq. (1) it can be seen that CTN decreases with $\left[\left(G^{2}+\right.\right.$ 1) $\left./ Y_{\text {coat }}\right]^{1 / 2}$, in which $G=Y_{\text {coat }} / Y_{\text {sub }}$. Therefore, it is lower for

\footnotetext{
${ }^{1}$ For a more detailed discussion of this approximation, see [15].
} 


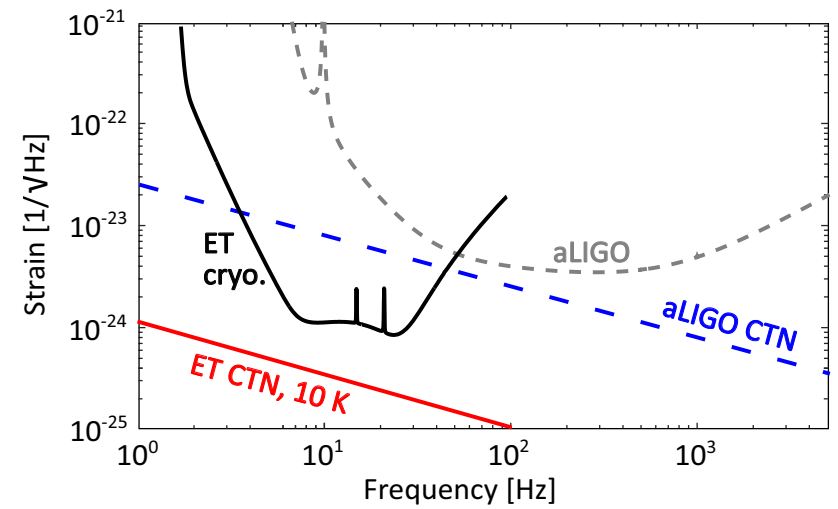

FIG. 1. Design sensitivity (gray, dashed curve) and CTN of Advanced LIGO (aLIGO) and design sensitivity and CTN at $10 \mathrm{~K}$ of the cryogenic Einstein Telescope (ET).

a large $Y_{\text {coat }}$, and in the case where $Y_{\text {sub }}>Y_{\text {coat }}$. However, as mechanical loss values of different materials generally differ much more than their Young's moduli, $\phi$ has proven to be the property mainly determining material selection. $d$ and $w$ are mainly determined by the interferometric design of the observatory. $w$ is limited by the available mirror substrate size and the ability to coat substrates of these dimensions with suitable quality, while $d$ depends on the required reflectivity.

The proportionality to $\sqrt{T}$ makes cooling the mirrors a very promising way forward to reduce CTN and therefore to improve sensitivity. This approach will be used in the Japanese KAGRA detector [16], which is currently under construction, in the planned European Einstein Telescope [17] and is also under consideration for future LIGO detectors.

In addition to low CTN, a low optical absorption is a highly relevant parameter for coating material selection. Heating due to absorption can cause difficulties in maintaining the low mirror temperature and lead to unwanted effects such as thermal lensing.

All these criteria make it challenging to find a coating meeting the requirements of the Einstein Telescope, which is designed to be $>10 \times$ more sensitive than current detectors. Recently, we suggested a suitable coating, using a multimaterial design which involves $\mathrm{HfO}_{2}$, doped with $\mathrm{SiO}_{2}$, and amorphous silicon ( $a$-Si) in addition to the commonly used coating materials $\mathrm{SiO}_{2}$ and $\mathrm{Ta}_{2} \mathrm{O}_{5}$ [18].

In their recent paper, Hasegawa et al. present observations of an ice layer growing on a mirror inside the cryogenic system of the KAGRA detector and a resulting change in the mirror's optical performance [19]. Here, we investigate the effect such an ice layer would have on CTN in the cryogenic Einstein Telescope.

\section{COATING THERMAL NOISE IN THE EINSTEIN TELESCOPE}

The Advanced LIGO and Advanced Virgo gravitational wave detectors operate at a wavelength of $1064 \mathrm{~nm}$ and at room temperature. They use fused silica $\left(\mathrm{SiO}_{2}\right)$ mirror substrates coated with $\mathrm{SiO}_{2}$ and $\mathrm{Ta}_{2} \mathrm{O}_{5}$ doped with $\mathrm{TiO}_{2}$. Figure 1 shows the design sensitivity of the Advanced LIGO detectors (gray, dashed curve) and the contribution of CTN (blue, dashed line). In the design study for the Einstein Telescope, an operation temperature of $10 \mathrm{~K}$ was suggested to reduce thermal noise at low detection frequencies [17]. The black solid curve in Fig. 1 shows the design sensitivity of the cryogenic Einstein Telescope with the design CTN at $10 \mathrm{~K}$ shown by the red, solid line. As higher temperatures around 20 and $120 \mathrm{~K}$ may also be of interest for the cryogenic Einstein Telescope, the effect of ice growth on CTN at these temperatures will also be discussed in this paper.

The mechanical loss of bulk fused silica increases by several orders of magnitude when being cooled to this temperature range [20,21], and a replacement mirror-substrate material is therefore required. For the Einstein Telescope, the use of crystalline silicon $(c-\mathrm{Si})$ is planned, which shows suitably low mechanical loss at low temperatures [22,23].

As $c$-Si shows high optical absorption at $1064 \mathrm{~nm}$, which decreases toward higher wavelengths [24,25], an increase in laser wavelength to $1550 \mathrm{~nm}$ is planned for the cryogenic Einstein Telescope. This results in increased CTN due to thicker coating layers. However, the larger Young's modulus of $c$-Si compared to fused silica has the inverse effect. A planned increase in laser beam diameter by a factor of 1.5 compared to Advanced LIGO is another factor reducing CTN.

The mechanical losses of the currently used coating materials $\mathrm{SiO}_{2}$ and $\mathrm{Ta}_{2} \mathrm{O}_{5}$ have been observed to increase at low temperatures compared to their room-temperature values as shown in Table I [26-28], resulting in less reduction in CTN than would be obtained from the temperature decrease alone.

In summary, all these factors (the favorable increase in $Y$ and $w$ and reduction in $T$, and the unfavorable increase in $d$ and $\phi$ ) would result in CTN being about a factor of 2 above the Einstein Telescope requirement (the red line in Fig. 1).

TABLE I. Material parameters for thermal-noise calculations. The heat treatment temperatures and sources of the loss values are explained in detail in the Appendix. It should be noted that some of the loss values for ice are based on extrapolation from directly measured numbers.

\begin{tabular}{|c|c|c|c|c|c|}
\hline & $\mathrm{SiO}_{2}$ & $\mathrm{Ta}_{2} \mathrm{O}_{5}$ & $a-\mathrm{Si}$ & $\mathrm{SiO}_{2}: \mathrm{HfO}_{2}$ & $a \mathrm{H}_{2} \mathrm{O}$ \\
\hline Young's modulus $Y(\mathrm{GPa})$ & $72[35]$ & $140[35]$ & $147[36]$ & $180[37]$ & $3.64[38]$ \\
\hline Refractive index $n$ at $1550 \mathrm{~nm}$ & $1.45[39]$ & $2.05[40]$ & $3.48[41]$ & $1.91[37]$ & $1.26^{\mathrm{a}}[19]$ \\
\hline \multicolumn{6}{|c|}{ Mechanical loss $\phi \times 10^{-4}$ at different temperatures } \\
\hline $300 \mathrm{~K}$ & $0.46[42]$ & $2.3[32]$ & $0.17[29]$ & & \\
\hline $120 \mathrm{~K}$ & $4.8[43]$ & $5.7[44]$ & $\leqslant 0.17[29]$ & $5.7[18]$ & $14[45](4.7)$ \\
\hline $20 \mathrm{~K}$ & $9.2[43]$ & $5.6[44]$ & $\leqslant 0.17[29]$ & $3.5[18]$ & $10(1)[38]$ \\
\hline $10 \mathrm{~K}$ & $8.5[43]$ & $5[44]$ & $\leqslant 0.17[29]$ & $3.7[18]$ & $7(1)[38]$ \\
\hline
\end{tabular}

${ }^{\mathrm{a}}$ For $1064 \mathrm{~nm}$. 


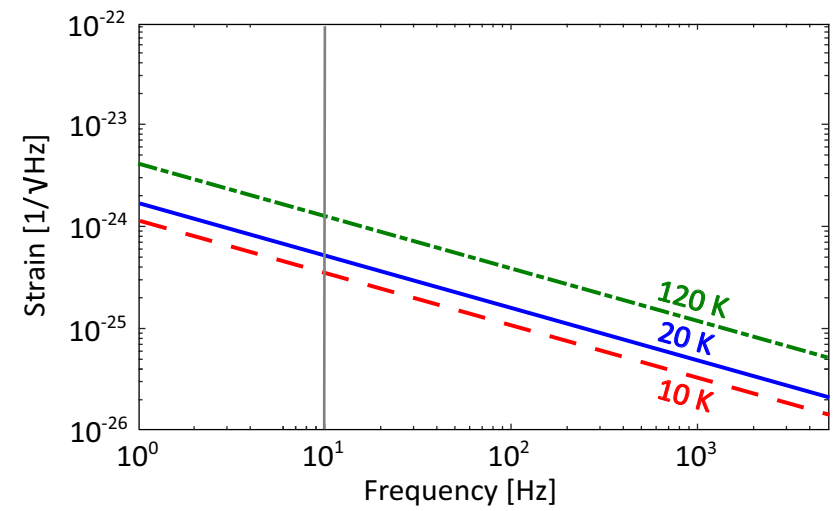

FIG. 2. CTN of the multimaterial coating presented in [18] when used for all four of the detectors arm-cavitiy mirrors, at temperatures of $10 \mathrm{~K}$ (bottom, red line), $20 \mathrm{~K}$ (middle, blue line), and $120 \mathrm{~K}$ (top, green line). The $10-\mathrm{K}$ line is identical to the red line in Fig. 1. The vertical, gray line marks the reference frequency of $10 \mathrm{~Hz}$ discussed throughout this paper.

To match the CTN design of the Einstein Telescope, a change in coating materials is required. The requirement that the optical absorption is $<5 \mathrm{ppm}$ makes development of suitable materials even more challenging. Materials with low mechanical loss such as $a$-Si $[29,30]$ and silicon nitride (SiN) [30,31] are promising, but show too high optical absorption. Different options for doping low-absorbing $\mathrm{Ta}_{2} \mathrm{O}_{5}$ are also under investigation [32,33], but so far could not match the thermal-noise requirements. Combining low-absorbing $\mathrm{SiO}_{2}$ and $\mathrm{Ta}_{2} \mathrm{O}_{5}$ with low-loss $a$-Si and $\mathrm{SiO}_{2}: \mathrm{HfO}_{2}$ in a multimaterial design [11,34] is so far the only option using amorphous materials to meet both the optical and thermalnoise requirements at $10 \mathrm{~K}$ stated in the Einstein Telescope Design Study [17,18].

Throughout this paper, we will discuss CTN using the example of this multimaterial coating. Figure 2 shows CTN for this coating at temperatures of $10 \mathrm{~K}$ (red, bottom line, identical with the red line in Fig. 1), $20 \mathrm{~K}$ (blue, middle line), and $120 \mathrm{~K}$ (green, top line). Mechanical losses and Young's moduli used for calculations are shown in Table I. The effect of an ice layer can be different when growing on a different coating with similar thermal noise. However, very likely the trends shown here will be representative for other coatings.

\section{COATING THERMAL NOISE OF ICE}

In this section we discuss the effect an ice layer would have on CTN of the mirrors in the Einstein Telescope.

\section{A. Ice growth on cryogenic mirrors}

In their paper, Hasegawa et al. present observations of an ice layer growing on a cryogenic mirror in the Japanese KAGRA detector [19]. The ice grows due to the flow of molecules from the room-temperature parts of the vacuum system to the cryogenic vacuum ducts where the test masses are located, where they are adsorbed onto the cold mirror surface. The residual gas molecules were identified with a mass spectrometer to be mainly $\mathrm{H}_{2} \mathrm{O}$, along with $\mathrm{O}$ and $\mathrm{OH}$, which can be generated from $\mathrm{H}_{2} \mathrm{O}$.

The reflectivity of the mirror in KAGRA changed periodically as the ice layer grew through multiples of quarterwavelengths in optical thickness. In addition, there was a longterm decreasing trend in reflectivity due to optical absorption in the ice layer. As a result, the circulating laser power in the interferometer decreased over time, with an oscillation superimposed on top of this decrease from the periodic reflectivity change. A change in power affects the detector sensitivity differently at different detection frequencies as explained in detail by Hasegawa et al. In general, a power decrease results in a shot-noise increase at high frequencies, while at low frequencies, at which also CTN is a significant noise source, radiation pressure noise decreases.

By monitoring the optical performance of the mirror, Hasegawa et al. observed a continuous layer growth over several weeks and extrapolated to the expected effect on the detector due to that additional, growing ice layer over a year. They conclude a growth rate of $(27 \pm 2) \mathrm{nm}$ /day for a mirror temperature of $47 \mathrm{~K}$. They also present a theoretical model for the growth rate of the ice layer. To obtain the conductance of the complex vacuum system, they use a Monte Carlo simulation. The resulting growth rate $\eta$ of the ice layer is 42 $\mathrm{nm} /$ day at $47 \mathrm{~K}$. The calculations we want to make here for the Einstein Telescope will be based on the experimentally obtained growth rate in KAGRA of $\eta=(27 \pm 2) \mathrm{nm} /$ day.

Here, we use literature values for the temperaturedependent mechanical loss of ice for calculating CTN. The material parameters were taken from [38] (see Table I) where the mechanical loss of ice is discussed in detail for different growth temperatures. More detail about the losses we chose to use in our calculations can be found in the Appendix of this paper. We find that an ice layer growing at the rate observed in KAGRA results in a significant increase in CTN dominated by the ice after only a few days of growth. The increasing trend of CTN due to ice is also superimposed by an oscillation, which is caused by the changing light field in the ice layer and amplified by the low Young's modulus of ice. In the following sections, we discuss these two effects in detail.

\section{B. Oscillation of coating thermal noise due to ice growth}

In a first-order approximation, coating thermal noise increases continuously with the coating thickness as shown by Eq. (1). However, when looking at a more detailed description of coating thermal noise, we find that layers show different contributions to the total coating thermal noise depending on their position in the coating stack and on their exact optical thickness, as the light field inside the coating changes due to interference effects $[12,46-48]$. Due to the continuous growth, the thermal-noise contribution of the layer oscillates, the maxima (and minima) separated by an optical thickness of a half-wavelength $\left(n \times d_{1}=\lambda / 2\right)$.

A more detailed description of coating thermal noise is given by [11]

$$
S_{x}(f)=\frac{2 k_{B} T}{\pi^{2} f} \frac{1}{w^{2}} \frac{1-\sigma_{\mathrm{sub}}-2 \sigma_{\mathrm{sub}}^{2}}{Y_{\mathrm{sub}}} \sum_{j} b_{j} d_{j} \phi_{j}
$$


$b_{j}$ is a weighting factor described by

$b_{j}=\frac{1}{1-\sigma_{j}}[\underbrace{\left(1-n_{j} \frac{\partial \theta_{\text {coat }}}{\partial \theta_{j}}\right)^{2}}_{\mathrm{A}} \underbrace{\frac{Y_{\text {sub }}}{Y_{j}}}_{\mathrm{B}}+\underbrace{\left(1-2 \sigma_{j}\right) \frac{Y_{j}}{Y_{\text {sub }}}}_{\mathrm{C}}]$

for $\sigma_{j} \approx \sigma_{\text {sub. }}$. The index $j$ refers to the material parameters defined in Eq. (1) for the $j$ th layer in the coating (starting from the outermost layer). The index sub refers to the substrate material parameters.

The second term of Eq. (3), marked as term $C$, describes the effect of coating thermal stresses driving the coating-tosubstrate interface [12]. This term remains constant as the ice layer grows. The first term of Eq. (3), given by $A \times B$, describes thermal noise arising from fluctuations in coating thickness. This is composed of two effects: a fluctuation in a layer changing (1) the optical thickness of this layer, and (2) the position of the front surface of the mirror. ${ }^{2}$ These two effects work in opposite directions and partly compensate. The first effect leads to fluctuations in the round-trip phase $\theta_{j}$ within the $j$ th coating layer. The term $\partial \theta_{\text {coat }} / \partial \theta_{j}$ describes the sensitivity of the total coating phase $\theta_{\text {coat }}$ to these fluctuations. The magnitude of $\partial \theta_{\text {coat }} / \partial \theta_{j}$ is proportional to the peak lightfield intensity in the $j$ th layer. As in HR coatings, the light field reduces with each double layer, the first effect is smaller for layers positioned further down in the coating. The second effect is independent of the layer position. Consequently, in lower layers, the first effect compensates less for the second effect and results in a stronger contribution of these layers to $b_{j}$, and therefore to the overall coating thermal noise. In the case of a growing ice layer, the coating phase sensitivity term oscillates due to the changing electric field intensity in the layer. $^{3}$

Figure 3 shows the different components of Eq. (3) for a better visualization of their contributions. In addition to the case of a growing ice layer, a $\mathrm{SiO}_{2}$ layer with identical optical thickness is shown for comparison. Figure 3(a) shows the coating phase sensitivity $\partial \theta_{\text {coat }} / \partial \theta_{j}$ (upper two curves), which oscillates with a period of $\lambda / 2$. The amplitude of this oscillation, which is proportional to the peak electric field intensity in the layer, is slightly bigger for $\mathrm{SiO}_{2}$ than for ice due to the higher refractive index. The two bottom curves in Fig. 3(a) show the part of Eq. (3) labeled $A$. The curves are still very similar for the two materials. Figure 3(b) shows the contributions of the oscillation term $A \times B$ and the constant term $C$ to the total weighting factor $b_{j}$ for $\mathrm{SiO}_{2}$. Figure 3(c) shows the same for ice. (Note the different $y$ axis.) It can be seen that the ratio of the Young's moduli $B$ causes the oscillation of the ice to be much stronger than for $\mathrm{SiO}_{2}$ due to the very small Young's modulus of ice. The second effect of this small Young's modulus is the $C$ term being significantly smaller for ice than for $\mathrm{SiO}_{2}$.

${ }^{2}$ In the simplified description of coating thermal noise [12,15] [see Eq. (1)], only the first effect is considered. Here, the penetration of the light field into the coating is also taken into account.

${ }^{3}$ While the reflectivity of the ETM changes only insignificantly $(<1 \%)$ as the ice layer grows on top, the amplitude of the light field within the ice layer changes by about $60 \%$ between the minimum (for multiples of $\lambda / 2$ ) and the maximum (for uneven multiples of $\lambda / 4$ ).

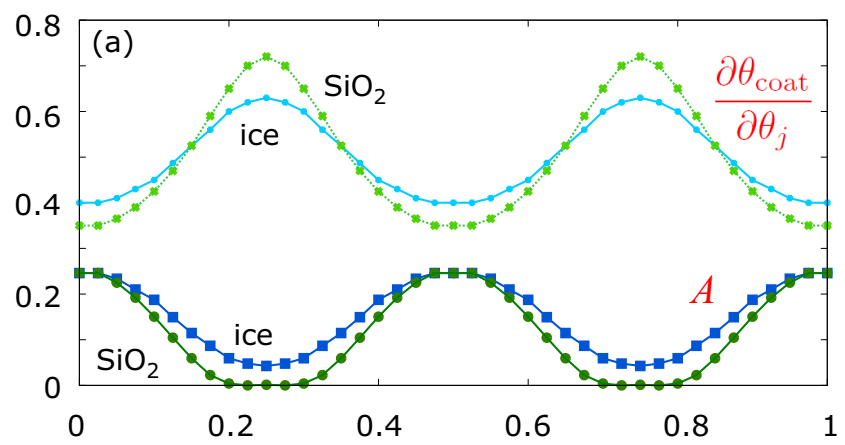

(b)

(c)

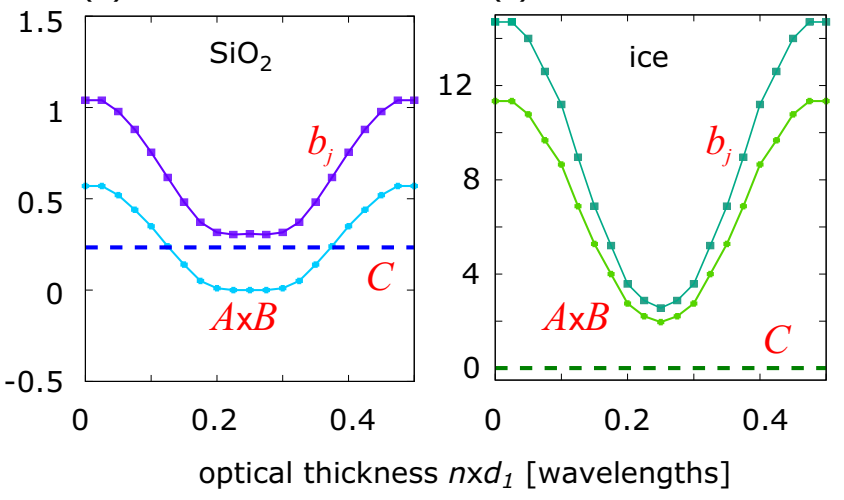

FIG. 3. Different components of Eq. (3). (a) Shows the coating phase sensitivity (two top curves) with increasing thickness for an ice layer on top of an ETM coating and $\mathrm{SiO}_{2}$ layer on top of the same coating. The two bottom curves show the $A$ term for both materials. (b) Shows the contributions of the oscillation term $A \times B$ and the constant term $C$ to the weighting term $b_{j}$ for $\mathrm{SiO}_{2}$. (c) Shows the equivalent terms for ice. (Note the different $y$ axis.)

To illustrate the effect of different material properties of the thermal-noise oscillation, it is instructive to imagine three cases of a material growing on top of an HR stack: ice, $\mathrm{SiO}_{2}$, and a material with the Young's modulus of $\mathrm{SiO}_{2}$ and all other properties identical to those of ice. Figure 4 shows coating thermal noise of an ETM coating calculated using Eq. (2) as a

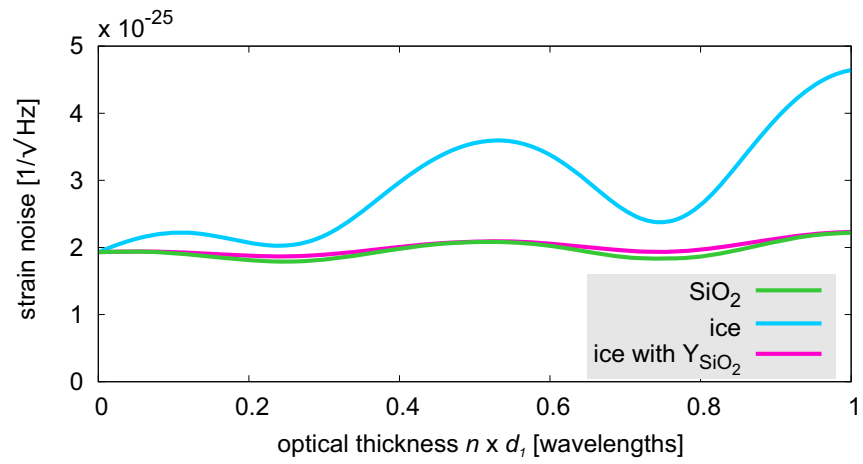

FIG. 4. CTN of an ETM coating at $10 \mathrm{~K}$ and a reference frequency of $10 \mathrm{~Hz}$. The $x$ axis shows the optical thickness $n \times l$ of a top layer in units of wavelengths $(\lambda)$. The green, bottom curve shows CTN for a cap layer made of $\mathrm{SiO}_{2}$ and the blue, top curve for a top layer made of ice. When assuming a Young's modulus identical to $\mathrm{SiO}_{2}$ for ice, the oscillation gets significantly weaker as shown by the pink curve (almost identical to the bottom curve for $\mathrm{SiO}_{2}$ ). 


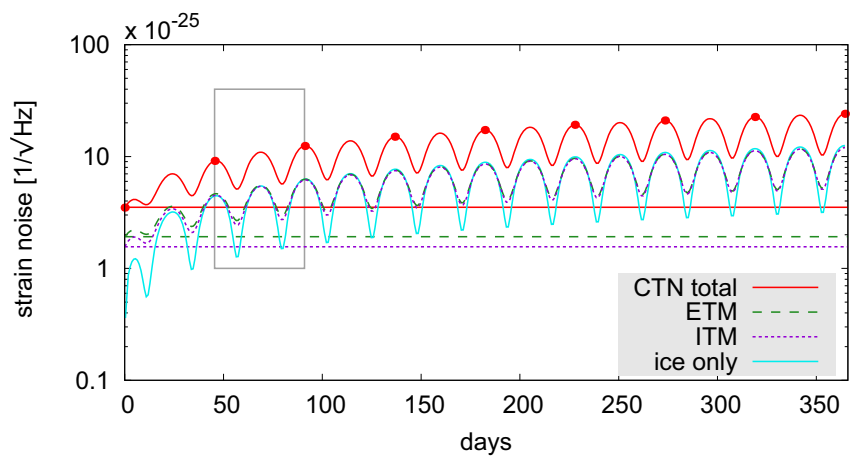

FIG. 5. Constant lines: CTN of the multimaterial coating presented in [18], separately for the ITM (bottom) and the ETM (middle), and when used for all four of the detectors arm-cavity mirrors (top), at a temperature of $10 \mathrm{~K}$ and a reference frequency of $10 \mathrm{~Hz}$. Oscillating curves: CTN of an ice layer only when growing on a $c$-Si substrate (bottom), separately on an ITM and an ETM (middle, almost identical) and total detector CTN (all four arm-cavity mirrors, top). Red dots on the total detector CTN mark thicknesses of ice at which $n \times d_{1}$ is an integer multiple of a full wavelength $\lambda(=1550 \mathrm{~nm})$.

function of the thickness of the first layer. When the first layer is ice, a large amplitude of thermal noise oscillation is seen (blue, top line). The overall increasing trend in thermal noise arises from the increasing $d$ [see Eq. (2)]. Next we consider the top layer to be made of $\mathrm{SiO}_{2}$ (green, bottom line), as an example of well-known amorphous coating material with a more typical Young's modulus than ice. In this case, the oscillation amplitude is much smaller. This reduced amplitude is almost entirely due to the higher Young's modulus of silica compared to ice. This is shown by the pink line, which shows the effect for an imaginary material with the Young's modulus of $\mathrm{SiO}_{2}$, but with all other properties identical to those of ice. This change in Young's modulus from the extremely low value of ice to a value closer to that of the substrate has an almost identical effect to that of an $\mathrm{SiO}_{2}$ layer, with only small differences caused by a slightly different $n$ and $\phi$ (see Table I).

\section{Increase of coating thermal noise with time}

The Einstein Telescope will have arm cavities with input test masses (ITMs) of reflectivity $R \approx 99.3 \%$ and ETMs of $R \approx 99.9995 \%$. The total CTN of the detector is given by $\left[2 \times\left(\mathrm{CTN}_{\mathrm{ETM}}{ }^{2}+\mathrm{CTN}_{\mathrm{ITM}^{2}}\right)\right]^{1 / 2}$, arising from two of each type of mirror. This total CTN is shown in Fig. 5 as a function of time at $10 \mathrm{~K}$ (constant, solid red line) for a reference frequency of $10 \mathrm{~Hz}$ (marked by the perpendicular, thick gray line in Fig. 2). The two bottom lines show CTN for the thinner ITM coating (purple, short dashes) and for the thicker ETM coating (green, long dashes). These three lines are flat as CTN is time independent.

The oscillating, blue line in Fig. 5 shows the CTN of an ice layer only ${ }^{4}\left(\phi_{10 \mathrm{~K}}=7 \times 10^{-4}\right)$, growing on a $c$-Si mirror

\footnotetext{
${ }^{4}$ To get an estimate of the effect of CTN of an ice layer only, we assumed lossless coating materials with Young's moduli identical to the $c$-Si substrate.
}

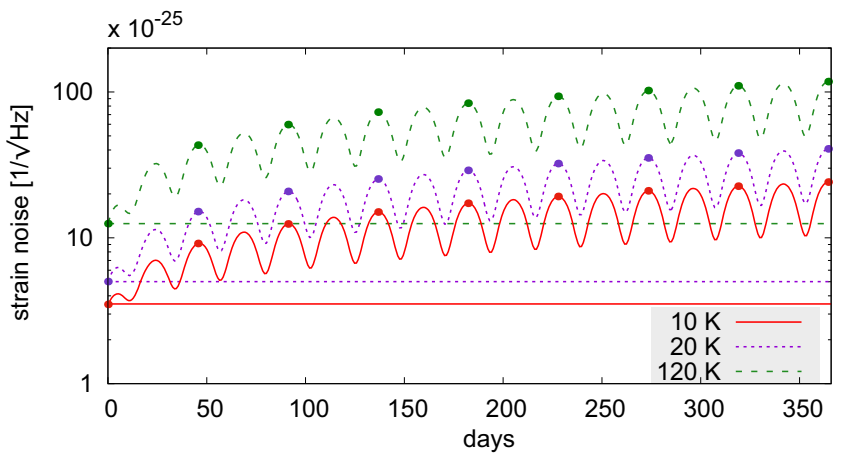

FIG. 6. Total detector CTN over one year at a reference frequency of $10 \mathrm{~Hz}$ at temperatures of $10 \mathrm{~K}$ (red, solid line), $20 \mathrm{~K}$ (blue, dotted line), and $120 \mathrm{~K}$ (green, dashed line). The oscillating curves show CTN with an ice layer growing on the mirrors, while the constant lines show CTN without ice. The 10-K curve and line are identical to the red curve and line in Fig. 5. Points on the oscillation represent thicknesses of ice at which $n \times d_{1}$ is an integer multiple of $\lambda$.

substrate at a rate of $27 \mathrm{~nm} /$ day. The oscillating green and purple dashed lines in Fig. 5 show CTN for an ETM and an ITM with a growing ice layer. The oscillation due to the ice layer dominates the shape of the (almost identical) ETM and ITM curves. The oscillating red, top curve shows CTN of the whole detector when ice grows on all four test-mass mirrors at the same rate. Red dots mark thicknesses of ice at which $n \times d_{1}$ is an integer multiple of a full wavelength $\lambda$; the gray rectangle marks one full layer optical thickness of ice grown. It can be observed that the oscillating curves with ice layers show significantly higher thermal noise, which increases as the ice layers grow, compared to the CTN without ice growth (flat bottom lines).

Figure 6 compares the total detector CTN including ice layers grown over one year at a reference frequency of $10 \mathrm{~Hz}$ at different temperatures. The red, solid line and curve $(10 \mathrm{~K})$ are identical to the red line and curve in Fig. 5. The blue, dotted and the green, dashed lines and curves show CTN at 20 and $120 \mathrm{~K}$ using losses for ice of $\phi=10 \times 10^{-4}$ and $14 \times 10^{-4}$ (see Table I). The points represent ice thicknesses for which $n \times d_{1}$ is an integer multiple of $\lambda$.

Table II shows the total detector CTN due to ice growing on the mirrors and the factor of CTN increase in brackets. Shown are the maximum increase during the first 30 days and during one year of detector operation, and the day at which this maximum occurs. Due to the lower growth rate, the increase in CTN is significantly lower for higher temperatures. However, the total CTN is still lowest at a temperature of $10 \mathrm{~K}$.

\section{REDUCTION OF COATING THERMAL NOISE FROM ICE}

In this section we will discuss ways to reduce the CTN increase occurring due to ice growth.

\section{A. Reduction via annealing}

The mechanical loss of ice reduces with annealing (see Table I). Heating the ice grown at $10 \mathrm{~K}$ to a temperature of $152 \mathrm{~K}$ can reduce the mechanical loss from $\phi=7 \times 10^{-4}$ 
TABLE II. Total detector CTN at a reference frequency of $10 \mathrm{~Hz}$ with and without ice at the different temperatures discussed. Given are the maximum increase during the first 30 days and during one year of detector operation, and the day at which CTN maximizes. The maximum CTN and corresponding days are also given for a reduced ice growth rate by a factor of 50 and for annealing of the ice every 91 days.

\begin{tabular}{|c|c|c|c|c|}
\hline $\mathrm{CTN} \times 10^{-25}$ & $(1 / \sqrt{H z})$ & $10 \mathrm{~K}$ & $20 \mathrm{~K}$ & $120 \mathrm{~K}$ \\
\hline No ice & & 3.5 & 5.0 & 12.5 \\
\hline \multicolumn{5}{|c|}{ Ice (with factor of increase shown in brackets) (see Fig. 6) } \\
\hline First 30 days & $\max$ & $7.0(2.0)$ & $11.4(2.3)$ & $32.3(2.6)$ \\
\hline Day & & & 25 & \\
\hline One year & $\max$ & $24.1(6.9)$ & $40.7(8.1)$ & $117.8(9.4)$ \\
\hline Day & & & 365 & \\
\hline \multicolumn{5}{|c|}{ Annealed ice (see Fig. 7 for $10 \mathrm{~K}$ ) } \\
\hline One year & $\max$ & $14.4(4.1)$ & $22.1(4.4)$ & $78.9(6.3)$ \\
\hline Day & & & 365 & \\
\hline \multicolumn{5}{|c|}{ Low growth rate (see Fig. 8) } \\
\hline One year & $\max$ & $4.1(1.2)$ & $6.3(1.3)$ & $16.7(1.3)$ \\
\hline Day & & 248 & 253 & 262 \\
\hline
\end{tabular}

to $1 \times 10^{-4}$ [38]. At $20 \mathrm{~K}$, a factor of about 10 reduction in loss might be possible, while at $120 \mathrm{~K}$ it is a factor of 3 (see Appendix for details).

Figure 7 shows the total detector CTN at $10 \mathrm{~K}$ for annealing the mirrors to $152 \mathrm{~K}$ every $91(\approx 3$ months) days (dashed curve). Here, we imagine heating the cryogenic mirror up to $152 \mathrm{~K}$ for a few hours to reduce the loss of the ice. For comparison, filled circles mark maxima of thermal noise without annealing (identical to the red dots in Fig. 6). The horizontal line marks CTN without ice growth. With this periodic annealing, the maximum CTN increase within a year is a factor of 4.1 , compared to a factor of 6.9 without annealing. Note that in this graph, we neglect the time it would take to heat up the mirror to $152 \mathrm{~K}$ and cool it back down to $10 \mathrm{~K}$, which would mean a significant interruption to the detector's observation

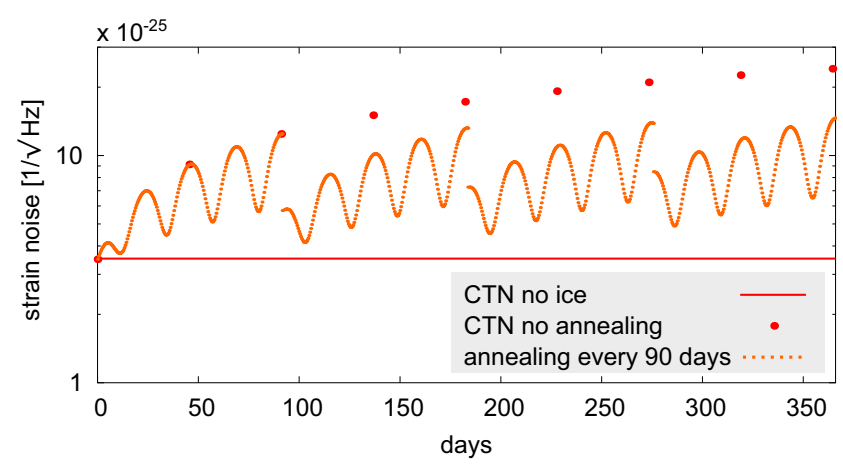

FIG. 7. Effect of annealing on the total detector CTN at $10 \mathrm{~K}$ and a reference frequency of $10 \mathrm{~Hz}$. The red, horizontal line shows CTN without ice. The dotted orange line shows CTN with ice growth. For the first 91 days, it is identical to the red, oscillating line in Fig. 6. Every 91 days (equivalent to an ice thickness of 2 optical layers $\lambda / n$ ), the loss of the ice was reduced (equivalent to $152-\mathrm{K}$ annealing). The red, filled circles mark thicknesses of unannealed ice for which $n \times d_{1}$ is an integer multiple of $\lambda$.

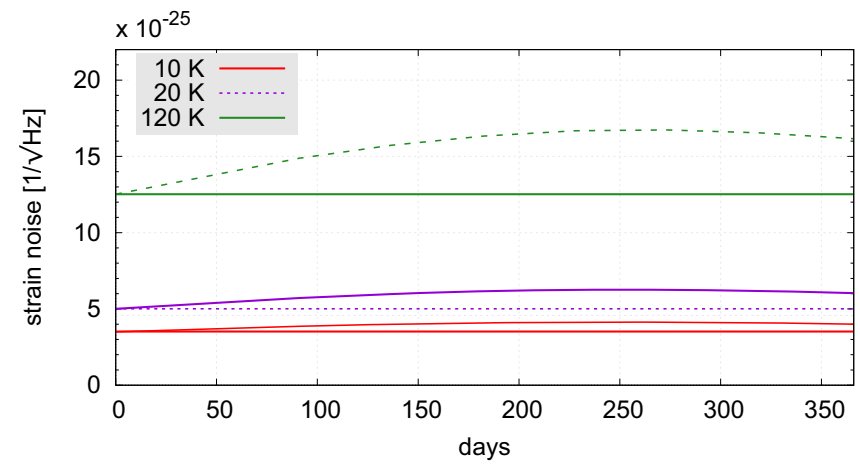

FIG. 8. Total detector CTN due to ice growth at different temperatures at a reference frequency of $10 \mathrm{~Hz}$ for an ice-growth rate a factor of 50 lower than in Fig. 6.

time. At 20 and at $120 \mathrm{~K}$ also a reduced CTN increase can be achieved from annealing (see Table II), with a higher increase due to the higher mechanical loss of ice at higher temperatures. However, for a detector operating temperature of $120 \mathrm{~K}$, heating and cooling the detector for an annealing period would take significantly less time and therefore seems to be more realistic than at lower temperatures.

\section{B. Reduction via growth-rate decrease}

Another way to reduce CTN increase due to ice growth is to significantly reduce the growth rate. As stated by Hasegawa et al., for KAGRA it is expected that the ice growth rate will reduce by up to a factor of 50 when reaching design pressure. Figure 8 shows the total detector CTN at different temperatures for a factor of 50 lower growth rate compared to Fig. 5 . For this lower growth rate, the maximum increase in CTN within a year compared to no ice growth is also presented in Table. II. The maximum increase is a factor of 1.2 at $10 \mathrm{~K}$ and 1.3 at 20 and $120 \mathrm{~K}$, which may be almost acceptable.

At this lower growth rate, a decrease in CTN due to annealing is still possible. Figure 9 shows CTN at $120 \mathrm{~K}$ for ice growth without annealing (dotted, purple line). For

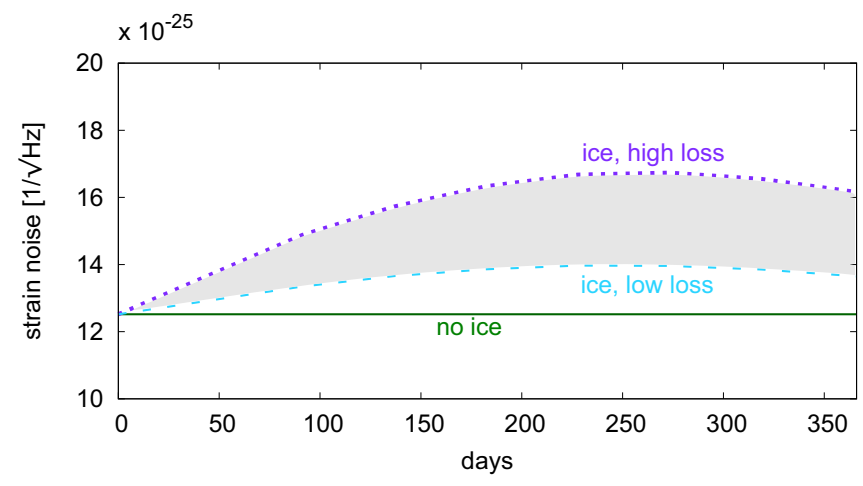

FIG. 9. Total detector CTN at $120 \mathrm{~K}$ without ice (solid, green line) and with ice growth (dotted, purple line) at a reference frequency of $10 \mathrm{~Hz}$; these lines are identical to the $120-\mathrm{K}$ data shown in Fig. 8. The dashed, light blue line shows the lower limit for CTN using the loss of annealed ice. Depending on how frequent annealing takes place, CTN will be within the gray area. 
comparison, in Fig. 9 the CTN without ice growth is also shown (solid, green line). These lines are identical to Fig. 8. For very frequent annealing, CTN would converge toward the level achievable for ice with the lower, annealed loss of $4.7 \times 10^{-4}$ as shown in Table I. This lower limit is given by the light blue, dashed line. Depending on the annealing frequency, which can be optimized for detector operation, CTN will be within the gray marked area.

\section{SUMMARY AND DISCUSSION}

We have theoretically investigated the effect an ice layer growing on the mirror surfaces could have on CTN in the Einstein Telescope based on the ice growth-rate observed in the KAGRA detector. It was found that after only a few days of growth, the ice layer will dominate CTN of the detector at operation temperatures of 10,20 , and $120 \mathrm{~K}$.

CTN tends to increase as the ice layer grows on top of the coatings. This increasing trend shows an oscillation associated with the changing optical thickness of the ice layer, with a high amplitude due to the very low Young's modulus of ice. The loss of ice is higher at higher temperatures (see Table I). As a result, CTN is highest at the highest operating temperature, agreeing with the trend observed without ice growth.

For the ice growth observed in KAGRA [19] and the mechanical losses found in the literature [38,45], CTN becomes intolerably high, up to a factor of 6.9 higher than the Einstein Telescope design value at $10 \mathrm{~K}$ within a year of operation, and up to a factor of 9.4 at $20 \mathrm{~K}$.

Annealing to about $152 \mathrm{~K}$ can reduce the mechanical loss of ice significantly $[38,45]$. Heating the mirrors to this temperature every 91 days would mean a reduced CTN increase (compared to no ice) to "only" a maximum of a factor of 4.1 at $10 \mathrm{~K}$, instead of a factor of 6.9 without annealing. However, it has to be noted that a temperature increase to $152 \mathrm{~K}$ and the following cooling back to operating temperature would mean a significant interruption to the detector's observation ability.

Hasegawa et al. expect the ice growth rate to be overestimated by up to a factor of 50 as KAGRA is not yet at design pressure. Therefore, we also estimated the CTN increase for a factor of 50 lower growth rate. In this case, the maximum CTN increase within one year of operation at $10 \mathrm{~K}$ would be only $20 \%$ above the Einstein Telescope design value.

At a potential operating temperature of $20 \mathrm{~K}$, the CTN increase due to ice is slightly higher than at $10 \mathrm{~K}$. Starting the annealing procedure from a base temperature of $20 \mathrm{~K}$ will not lead to significantly shorter interruptions in detector operation as when starting from $10 \mathrm{~K}$. Therefore, while an operating temperature of $20 \mathrm{~K}$ may be desirable for other reasons, there is no advantage over $10 \mathrm{~K}$ from an ice $\mathrm{CTN}$ perspective. However, at $120 \mathrm{~K}$, annealing to $152 \mathrm{~K}$ would mean only short interruptions to detector operation.

It should be noted that all these considerations are based on growth-rate measurements in the current, not yet finalized, KAGRA configuration, and on mechanical loss measurements from the literature. While parameters have been chosen to be as close to the Einstein Telescope parameters as possible, we suggest detailed studies of the expected ice growth for the Einstein Telescope configuration as well as of the CTN of HR mirror coatings with an ice layer on top.

\section{ACKNOWLEDGMENTS}

We are grateful for financial support from STFC (Grant No. ST/N005422/1) and the University of Glasgow. I.W.M. is supported by a Royal Society Research Fellowship. We are grateful to the International Max Planck Partnership for Measurement and Observation at the Quantum Limit for support, and we thank our colleagues in the LSC and Virgo collaborations and within Scottish Universities Physics Alliance (SUPA) for their interest in this work. We would like to thank Stefan Hild for his close reading and useful questions and Hans-Herbert Otto for inspiring this work. This paper has LIGO Document number LIGO-P1900168.

\section{APPENDIX: MECHANICAL LOSS OF ICE AND OPTICAL COATING MATERIALS}

Here, we describe the origin of all of the mechanical loss values given in Table I. The heat-treatment temperature of the multimaterial coating suggested in [18] will most likely be determined by the $a$-Si layers for absorption reasons: the optical absorption of $\mathrm{a}$-Si minimizes between $400{ }^{\circ} \mathrm{C}$ and $500{ }^{\circ} \mathrm{C}$, varying slightly with the exact deposition procedure. The mechanical loss for the other materials was chosen to be within this temperature range. However, data for identical heat treatment temperatures were not available for all materials.

$\mathrm{SiO}_{2}$. The loss of $\mathrm{SiO}_{2}$ films at room temperature was taken from [42], and is for a heat-treatment temperature of $500{ }^{\circ} \mathrm{C}$. The films were $500 \mathrm{~nm}$ thick and deposited by LMA (Laboratoire des Matériaux Avancés). The low-temperature loss values for $\mathrm{SiO}_{2}$ are for a heat-treatment temperature of $450{ }^{\circ} \mathrm{C}$ [43] for a 500-nm film deposited by ATF (Advanced Thin Films).

$\mathrm{Ta}_{2} \mathrm{O}_{5}$. The loss of $\mathrm{Ta}_{2} \mathrm{O}_{5}$ at room temperature is for a $\mathrm{Ta}_{2} \mathrm{O}_{5}$ film doped with $\mathrm{TiO}_{2}$ (14\% cation concentration), heat treated at $500{ }^{\circ} \mathrm{C}$ [42]. The film was $500 \mathrm{~nm}$ thick and was deposited by LMA. The cryogenic loss values of $\mathrm{Ta}_{2} \mathrm{O}_{5}$ are for a heat-treatment temperature of $400{ }^{\circ} \mathrm{C}$. Loss values of undoped $\mathrm{Ta}_{2} \mathrm{O}_{5}$ were used as no data for doped $\mathrm{Ta}_{2} \mathrm{O}_{5}$ were available for this heat-treatment temperature. The films were $500 \mathrm{~nm}$ in thickness and deposited by CSIRO (Commonwealth Scientific and Industrial Research Organization).

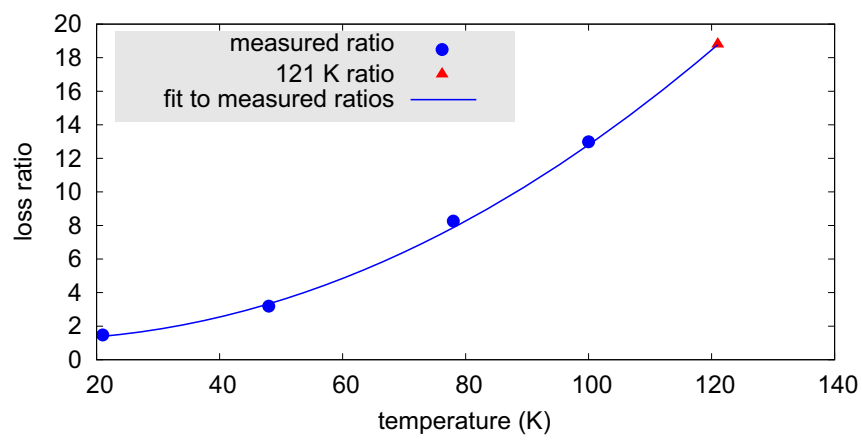

FIG. 10. Ratio of the loss of ice at deposition temperature to the loss of the same ice layer at $4 \mathrm{~K}$ operating temperature, as a function of deposition temperature based on data from [45]. Also shown is the fit used to extrapolate the expected ratio for a deposition temperature of $121 \mathrm{~K}$, and the extrapolated ratio value. 
$a-S i$. The loss of $a$-Si (deposited using electron cyclotron resonance ion-beam sputtering) was assumed to be $0.17 \times$ $10^{-4}$ at all temperatures. This value is taken from [29], where it was measured at room temperature for a film deposited at $200{ }^{\circ} \mathrm{C}$ and then heat treated at $400^{\circ} \mathrm{C}$. $a$-Si films tend to show a decreasing loss on cooling below room temperature [49]. Therefore, in the absence of data for this coating at cryogenic temperatures, we have used the room-temperature value as a likely upper limit on the cryogenic loss. For the material presented in [29], the optimum heat-treatment temperature to minimize the optical absorption was $450^{\circ} \mathrm{C}$. There is evidence of a decrease of the mechanical loss of $a$-Si for slightly higher heat-treatment temperatures than $400{ }^{\circ} \mathrm{C}[30,49]$. Therefore, an upper limit of $0.17 \times 10^{-4}$ for the loss is still valid.

$\mathrm{SiO}_{2}: \mathrm{HfO}_{2}$. The loss of $\mathrm{SiO}_{2}: \mathrm{HfO}_{2}$ was taken from [18] and was measured for a 500-nm-thick coating deposited by CSIRO and heat treated at $400{ }^{\circ} \mathrm{C}$. The $\mathrm{SiO}_{2}$ cation concentration was $27 \%$.

$a-H_{2} \mathrm{O}$. Hessinger et al. have measured the temperature dependence of the loss of amorphous ice layers grown at different temperatures $[38,45]$. For all growth temperatures, a plateau in loss was observed around $4 \mathrm{~K}$, with the loss tending to increase at higher measurement temperatures.

For an operating temperature of the gravitational wave detector of $20 \mathrm{~K}$, we have taken the loss value for ice grown at $21 \mathrm{~K}$ from Fig. 10 in [38]. No data were available for ice grown at $10 \mathrm{~K}$. Therefore, we have used the loss value for a growth temperature of $21 \mathrm{~K}$ (which was the closest to $10 \mathrm{~K}$ that was available in [38]), measured at $10-\mathrm{K}$ operating temperature.

There are no measurements of the loss of ice grown at $\sim 120 \mathrm{~K}$ in [38]. However, for ice layers grown at other temperatures, the ratio of the losses at deposition temperature and at $4 \mathrm{~K}$ was found to increase with deposition temperature. A fit to this relationship, shown in Fig. 10, was used to estimate the loss of ice grown at $\sim 120 \mathrm{~K}$.

For all three temperatures, a loss decrease from annealing can be estimated. For $20 \mathrm{~K}$, the effect has directly been measured [38]. We assume an identical loss as an upper limit at $10 \mathrm{~K}$. For $120 \mathrm{~K}$, a factor of 3 reduction due to annealing near deposition temperature was estimated from the data in [38].
[1] B. P. Abbott, R. Abbott, T. D. Abbott, M. R. Abernathy, F. Acernese, K. Ackley, C. Adams, T. Adams, P. Addesso, R. X. Adhikari et al., Observation of Gravitational Waves from a Binary Black Hole Merger, Phys. Rev. Lett. 116, 061102 (2016).

[2] A. Einstein, Approximative integration of the field equations of gravitation, Sitzungsber. Preuss. Akad. Wiss. Berlin 1916, 688 (1916).

[3] A. Einstein, Über Gravitationswellen, Sitzungsber. Preuss. Akad. Wiss. Berlin 1918, 154 (1918).

[4] B. P. Abbott, R. Abbott, T. D. Abbott, M. R. Abernathy, F. Acernese, K. Ackley, C. Adams, T. Adams, P. Addesso, R. X. Adhikari et al., GW151226: Observation of Gravitational Waves from a 22-Solar-Mass Binary Black Hole Coalescence, Phys. Rev. Lett. 116, 241103 (2016).

[5] B. P. Abbott, R. Abbott, T. D. Abbott, M. R. Abernathy, F. Acernese, K. Ackley, C. Adams, T. Adams, P. Addesso, R. X. Adhikari et al., GW170104: Observation of a 50-Solar-Mass Binary Black Hole Coalescence at Redshift 0.2, Phys. Rev. Lett. 118, 221101 (2017).

[6] B. P. Abbott, R. Abbott, T. D. Abbott, M. R. Abernathy, F. Acernese, K. Ackley, C. Adams, T. Adams, P. Addesso, R. X. Adhikari et al., GW170814: A Three-Detector Observation of Gravitational Waves from a Binary Black Hole Coalescence, Phys. Rev. Lett. 119, 141101 (2017).

[7] B. P. Abbott, R. Abbott, T. D. Abbott, M. R. Abernathy, F. Acernese, K. Ackley, C. Adams, T. Adams, P. Addesso, R. X. Adhikari et al., GW170817: Observation of Gravitational Waves from a Binary Neutron Star Inspiral, Phys. Rev. Lett. 119, 161101 (2017).

[8] B. P. Abbott, R. Abbott, T. D. Abbott, S. Abraham, F. Acernese, K. Ackley, C. Adams, R. X. Adhikari, V. B. Adya, C. Affeldt et al. (The LIGO Scientific and Virgo Collaborations), GWTC-1: A Gravitational-Wave Transient Catalog of Compact Binary Mergers Observed by LIGO and Virgo during the First and Second Observing Runs, arXiv:1811.12907 [Phys. Rev. X (to be published)].
[9] J. Aasi, B. P. Abbott, R. Abbott, T. Abbott, M. R. Abernathy, K. Ackley, C. Adams, T. Adams, P. Addesso, R. X. Adhikari et al., Advanced LIGO, Classical Quantum Gravity 32, 074001 (2015).

[10] F. Acernese, M. Agathos, K. Agatsuma, D. Aisa, N. Allemandou, A. Allocca, J. Amarni, P. Astone, G. Balestri, G. Ballardin et al., Advanced Virgo: a 2nd generation interferometric gravitational wave detector, Classical Quantum Gravity 32, 024001 (2015).

[11] W. Yam, S. Gras, and M. Evans, Multimaterial coatings with reduced thermal, Phys. Rev. D 91, 042002 (2015).

[12] T. Hong, H. Yang, E. K. Gustafson, R. X. Adhikari, and Y. Chen, Brownian thermal noise in multilayer coated mirrors, Phys. Rev. D 87, 082001 (2013).

[13] G. M. Harry, M. R. Abernathy, A. E. Becerra-Toledo, H. Armandula, E. Black, K. Dooley, M. Eichenfield, C. Nwabugwu, A. Villar, D. R. M. Crooks, G. Cagnoli, J. Hough, C. R. How, I. MacLaren, P. Murray, S. Reid, S. Rowan, P. H. Sneddon, M. M. Fejer, R. Route et al., Titania-doped tantala/silica coatings for gravitational-wave detection, Classical Quantum Gravity 24, 405 (2007).

[14] P. R. Saulson, Thermal noise in mechanical experiments, Phys. Rev. D 42, 2437 (1990).

[15] G. M. Harry, A. M. Gretarsson, P. R. Saulson, S. E. Kittelberger, S. D. Penn, W. J. Startin, S. Rowan, M. M. Fejer, D. R. M. Crooks, G. Cagnoli, J. Hough, and N. Nakagawa, Thermal noise in interferometric gravitational wave detectors due to dielectric optical coatings, Classical Quantum Gravity 19, 897 (2002).

[16] K. Somiya for the KAGRA collaboration, Detector configuration of KAGRA-the Japanese cryogenic gravitational-wave detector, Classical Quantum Gravity 29, 124007 (2012).

[17] M. Abernathy, F. Acernese, P. Ajith, B. Allen, P. AmaroSeoane, N. Andersson, S. Aoudia, P. Astone, B. Krishnan, L. Barack et al., Einstein gravitational wave Telescope (ET) conceptual design study, ET-0106C-10, https://tds.ego-gw.it/ql/ ?c=7954 (2010). 
[18] K. Craig, J. Steinlechner, P. G. Murray, A. S. Bell, R. Birney, K. Haughian, J. Hough, I. MacLaren, S. Penn, S. Reid, R. Robie, S. Rowan, and I. W. Martin, Mirror Coating Solution for the Cryogenic Einstein Telescope, Phys. Rev. Lett. 122, 231102 (2019).

[19] K. Hasegawa, T. Akutsu, N. Kimura, Y. Saito, T. Suzuki, T. Tomaru, A. Ueda, and S. Miyoki, Molecular adsorbed layer formation on cooled mirrors and its impacts on cryogenic gravitational wave telescopes, Phys. Rev. D 99, 022003 (2019).

[20] J. W. Marx and J. M. Sivertsen, Temperature dependence of the elastic moduli and internal friction of silica and glass, J. Appl. Phys. 24, 81 (1953).

[21] A. Schroeter, R. Nawrodt, R. Schnabel, S. Reid, I. Martin, S. Rowan, C. Schwarz, T. Koettig, R. Neubert, M. Thürk, W. Vodel, A. Tünnermann, K. Danzmann, and P. Seidel, On the mechanical quality factors of cryogenic test masses from fused silica and crystalline quartz, arXiv:0709.4359v1.

[22] D. F. McGuigan, C. C. Lam, R. Q. Gram, A. W. Hoffman, D. H. Douglass, and H. W. Gutche, Measurements of the mechanical Q of single-crystal silicon at low temperatures, J. Low Temp. Phys. 30, 624 (1978).

[23] R. Nawrodt, A. Zimmer, T. Koettig, C. Schwarz, D. Heinert, M. Hudl, R. Neubert, M. Thürk, S. Nietzsche, W. Vodel, P. Seidel, and A. Tünnermann, High mechanical Q-factor measurements on silicon bulk samples, J. Phys.: Conf. Ser. 122, 012008 (2008).

[24] M. J. Keevers and M. A. Green, Absorption edge of silicon from solar cell spectral response measurements, Appl. Phys. Lett. 66, 174 (1995).

[25] J. Degallaix, R. Flaminio, D. Forest, M. Granata, C. Michel, L. Pinard, T. Bertrand, and G. Cagnoli, Bulk optical absorption of high resistivity silicon at $1550 \mathrm{~nm}$, Opt. Lett. 38, 2047 (2013).

[26] M. Granata, K. Craig, G. Cagnoli, C. Carcy, W. Cunningham, J. Degallaix, R. Flaminio, D. Forest, M. Hart, J.-S. Hennig, J. Hough, I. MacLaren, I. W. Martin, C. Michel, N. Morgado, S. Otmani, L. Pinard, and S. Rowan, Cryogenic measurements of mechanical loss of high-reflectivity coating and estimation of thermal noise, Opt. Lett. 38, 5268 (2013).

[27] I. W. Martin, R. Nawrodt, K. Craig, C. Schwarz, R. Bassiri, G. Harry, J. Hough, S. Penn, S. Reid, R. Robie, and S. Rowan, Low temperature mechanical dissipation of an ion-beam sputtered silica film, Classical Quantum Gravity 31, 035019 (2014).

[28] I. W. Martin et al., Comparison of the temperature dependence of the mechanical dissipation in thin films of $\mathrm{Ta}_{2} \mathrm{O}_{5}$ and $\mathrm{Ta}_{2} \mathrm{O}_{5}$ doped with $\mathrm{TiO}_{2}$, Classical Quantum Gravity 26, 155012 (2009).

[29] R. Birney, J. Steinlechner, Z. Tornasi, S. MacFoy, D. Vine, A. S. Bell, D. Gibson, J. Hough, S. Rowan, P. Sortais, S. Sproules, S. Tait, I. W. Martin, and S. Reid, Amorphous Silicon with Extremely Low Absorption: Beating Thermal Noise in Gravitational Astronomy, Phys. Rev. Lett. 121, 191101 (2018).

[30] J. Steinlechner, I. W. Martin, A. S. Bell, J. Hough, M. Fletcher, P. G. Murray, R. Robie, S. Rowan, and R. Schnabel, SiliconBased Optical Mirror Coatings for Ultrahigh Precision Metrology and Sensing, Phys. Rev. Lett. 120, 263602 (2018).

[31] H. Pan, L. Kuo, L. Chang, S. Chao, I. Martin, J. Steinlechner, and M. Fletcher, Silicon nitride and silica quarter-wave stacks for low-thermal-noise mirror coatings, Phys. Rev. D 98, 102001 (2018).

[32] R. Flaminio, J. Franc, C. Michel, N. Morgado, L. Pinard, and B. Sassolas, A study of coating mechanical and optical losses in view of reducing mirror thermal noise in gravitational wave detectors, Classical Quantum Graity. 27, 084030 (2010).

[33] K. Prasai, J. Jiang, A. Mishkin, B. Shyam, S. Angelova, R. Birney, D. A. Drabold, M. Fazio, E. K. Gustafson, G. Harry, S. Hoback, J. Hough, C. Lévesque, I. MacLaren, A. Markosyan, I. W. Martin, C. S. Menoni, P. G. Murray, S. Penn, S. Reid et al., High Precision Detection of Change in Intermediate Range Order of Amorphous Zirconia-Doped Tantala Thin Films Due to Annealing, Phys. Rev. Lett. 123, 045501 (2019).

[34] J. Steinlechner, I. W. Martin, C. Krueger, J. Hough, S. Rowan, and R. Schnabel, Thermal noise reduction and absorption optimisation via multi-material coatings, Phys. Rev. D 91, 042001 (2015).

[35] M. M. Fejer, S. Rowan, G. Cagnoli, D. R. M. Crooks, A Gretarsson, G. M. Harry, J. Hough, S. D. Penn, P. H. Sneddon, and S. P. Vyatchanin, Thermoelastic dissipation in inhomogeneous media: loss measurements and displacement noise in coated test masses for interferometric gravitational wave detectors, Phys. Rev. D 70, 082003 (2004).

[36] M. R. Abernathy, Mechanical properties of coating materials for use in the mirrors of interferometric gravitational wave detectors, Ph.D. thesis, University of Glasgow, UK, 2012.

[37] K. Craig, Studies of the mechanical dissipation of thin films for mirrors in interferometric gravitational wave detectors, Ph.D. Thesis, University of Glasgow, UK, 2015.

[38] J. Hessinger, B. E. White Jr., and R. O. Pohl, Elastic properties of amorphous and crystalline ice films, Planet Space Sci. 44, 937 (1996).

[39] D. B. Leviton and B. J. Frey, Temperature-dependent absolute refractive index measurements of synthetic fused silica, Proc. SPIE 6273, 62732K (2006).

[40] J. Franc, N. Morgado, R. Flaminio, R. Nawrodt, I. Martin, L. Cunningham, A. Cumming, S. Rowan, and J. Hough, Mirror thermal noise in laser interferometer gravitational wave detectors operating at room and cryogenic temperature arXiv:0912.0107.

[41] D. T. Pierce and W. E. Spicer, Electronic structure of amorphous Si from photoemission and optical studies, Phys. Rev. B 5, 3017 (1972).

[42] M. Granata, E. Saracco, N. Morgado, A. Cajgfinger, G. Cagnoli, J. Degallaix, V. Dolique, D. Forest, J. Franc, C. Michel, L. Pinard, and R. Flaminio, Mechanical loss in state-of-theart amorphous optical coatings, Phys. Rev. D 93, 012007 (2016).

[43] R. R. Robie, Characterisation of the mechanical properties of thin-film mirror coating materials for use in future interferometric gravitational wave detectors, Ph.D. thesis, University of Glasgow, UK, 2018.

[44] I. W. Martin, R. Bassiri, R. Nawrodt, M. M. Fejer, A. Gretarsson, E. Gustafson, G. Harry, J. Hough, I. MacLaren, S. Penn, S. Reid, R. Route, S. Rowan, C. Schwarz, P. Seidel, J. Scott, and A. L. Woodcraft, Effect of heat treatment on mechanical dissipation in $\mathrm{Ta}_{2} \mathrm{O}_{5}$ coatings, Classical Quantum Gravity 27, 225020 (2010). 
[45] J. Hessinger and R. O. Pohl, Annealing of amorphous ice films, J. Non-Cryst. Solids 208, 151 (1996).

[46] M. L. Gorodetsky, Thermal noises and noise compensation in high-reflection multilayer coating, Phys. Lett. A 372, 6813 (2008).

[47] A. Gurkovsky and S. Vyatchanin, Thermal noises and noise compensation in high-reflection multilayer coating, Phys. Lett. A 374, 3267 (2010).
[48] N. M. Kondratiev, A. G. Gurkovsky, and M. L. Gorodetsky, Thermal noise and coating optimization in multilayer dielectric mirrors, Phys. Rev. D 84, 022001 (2011).

[49] P. G. Murray, I. W. Martin, K. Craig, J. Hough, R. Robie, S. Rowan, M. R. Abernathy, T. Pershing, and S. Penn, Ionbeam sputtered amorphous silicon films for cryogenic precision measurement systems, Phys. Rev. D 92, 062001 (2015). 